\title{
TWO-MACHINE FLOWSHOP SCHEDULING PROBLEM TO MINIMIZE MAKESPAN OR TOTAL COMPLETION TIME WITH RANDOM AND BOUNDED SETUP TIMES
}

\author{
ALI ALLAHVERDI, TARIQ ALDOWAISAN, and YURI N. SOTSKOV
}

\author{
Received 21 October 2002
}

\begin{abstract}
This paper addresses the two-machine flowshop scheduling problem with separate setup times to minimize makespan or total completion time (TCT). Setup times are relaxed to be random variables rather than deterministic as commonly used in the OR literature. Moreover, distribution-free setup times are used where only the lower and upper bounds are given. Global and local dominance relations are developed for the considered flowshops and an illustrative numerical example is given.
\end{abstract}

2000 Mathematics Subject Classification: 90B36, 90B35, 90B30.

1. Introduction. The two-machine flowshop scheduling problem is polynomially solvable when the objective is to minimize makespan, see [7]. The problem, however, is proven to be NP-hard when the performance measure is total completion time (TCT), see [6]. In these and the vast majority of subsequent research on the flowshop problems, it is assumed that either the setup time for a job is included in its processing time or the setup times are zero on each machine. While these assumptions may be justified for some real scheduling problems, other situations call for explicit (separate) setup time consideration. The practical situations in which setup times must be considered as separate include the chemical, pharmaceutical, printing, food processing, metal processing, and semiconductor industries, see [3] for a survey on the scheduling problems with separate setup times.

An obvious advantage of considering setup times separate is that when there exists idle time on the second machine (usually the case), then the setup time for a job on the second machine can be performed prior to the completion time of this job on the first machine. This means that the performance measures of makespan and TCT may be improved by considering setup times as separate from processing times. Hence, some researchers considered the flowshop problem with separate setup times, that is, relaxing the assumption that setup times are included in processing times. Yoshida and Hitomi [11], Khurana and Bagga [8], and Allahverdi [1] addressed the problem of minimizing makespan 
by considering setup times as separate. Bagga and Khurana [5] and Allahverdi [2], on the other hand, addressed the two-machine separate setup time problem with respect to TCT. In the research mentioned so far, the setup times are considered as separate from processing times but assumed to be deterministic, that is, known before scheduling and fixed during a realization of the process. In reality, this assumption is not valid in many cases, and setup times have to be considered as random variables. Moreover, it is sometimes difficult to find an appropriate probability distribution for random setup times.

In this paper, we address the two-machine flowshop scheduling problem to minimize TCT (sum of job completion times) or makespan (maximum of the job completion times) when assuming that deterministic setup times are not realistic and it is hard to obtain exact probability distributions for random setup times. As such, a solution obtained by assuming fixed setup times or by assuming a certain probability distribution may not be close to the optimal schedule for the realization of the process. It has been observed that although the exact probability distribution of setup times may not be known before scheduling, some upper and lower bounds on job setup times are easy to obtain in most practical cases. This information on the bounds of setup times is important, and it should be utilized in finding a solution for the scheduling problem.

We consider the scheduling environments in which setup times are random variables with unknown probability distributions when only a lower bound $L_{j, m} \geq 0$ and an upper bound $U_{j, m} \geq L_{j, m}$ of the setup time $S_{j, m}$ of job $j$ on machine $m$ are given before scheduling. Such a flowshop problem with two machines is denoted as $F 2\left|S_{j, m}, L_{j, m} \leq S_{j, m} \leq U_{j, m}\right| \sum C_{j}$ for TCT criterion and as $F 2\left|S_{j, m}, L_{j, m} \leq S_{j, m} \leq U_{j, m}\right| C_{\max }$ for the makespan criterion. Hereafter, $C_{j}$ denotes the completion time of job $j$ on the second machine and $C_{\max }$ denotes the makespan criterion. Notice that the problems $F 2\left|S_{j, m}, L_{j, m} \leq S_{j, m} \leq U_{j, m}\right| C_{\max }$ and $F 2\left|S_{j, m}, L_{j, m} \leq S_{j, m} \leq U_{j, m}\right| \sum C_{j}$ can be considered as stochastic flowshop problems under uncertainty of setup times when there is no prior information about probability distributions of the random setup times (it is only known that setup times will fall between the given lower and upper bounds with probability one). Similar problems have been addressed in the literature for the case where job processing times are random variables but setup times are assumed to be zero, see $[4,9,10]$.

If the equalities $L_{j, m}=S_{j, m}=U_{j, m}$ hold for each job $j \in J$ and each machine $m \in M$, where $J=\{1,2, \ldots, n\}$ denotes the set of jobs and $M=\{1,2\}$ denotes the set of machines, then the problem $F 2\left|S_{j, m}, L_{j, m} \leq S_{j, m} \leq U_{j, m}\right| \sum C_{j}$ reduces to the flowshop problem $F 2\left|S_{j, m}\right| \sum C_{j}$ with fixed setup times that was considered by Bagga and Khurana [5] and Allahverdi [2]. Similarly, the problem $F 2\left|S_{j, m}, L_{j, m} \leq S_{j, m} \leq U_{j, m}\right| C_{\max }$ becomes a deterministic flowshop problem $F 2\left|S_{j, m}\right| C_{\max }$ which is polynomially solvable if all setup times are fixed and sequence-independent, see [11]. Setup time is called sequence-independent if 
it depends only on the job to be processed. On the other hand, if the setup time depends on both the job to be processed and the immediately preceding job, then it is called sequence-dependent. When setup times are sequenceindependent on both machines, then permutation schedules are dominant with respect to any regular criterion, see [11]. Thus, in order to find an optimal schedule, one only needs to consider the same sequence of jobs on both machines. In the problems $F 2\left|S_{j, m}, L_{j, m} \leq S_{j, m} \leq U_{j, m}\right| C_{\max }$ and $F 2 \mid S_{j, m}, L_{j, m} \leq$ $S_{j, m} \leq U_{j, m} \mid \sum C_{j}$ under consideration, we assume that setup times are sequence-independent on both machines, and hence, permutation schedules are dominant with respect to any regular criterion.

The rest of this paper is organized as follows. The main notations are given in Section 2. Global and local dominance relations are established in Sections 3 and 4 for both makespan and TCT criteria. Section 5 presents illustrative examples and Section 6 provides concluding remarks.

2. Definitions and notations. Let $P_{j, m}$ denote the processing time of job $j(j \in J=\{1,2, \ldots, n\})$ on machine $m(m \in M=\{1,2\})$ and let the bracket $[i, m]$ denote the job in position $i$ on machine $m$. That is, $P_{[i, m]}$ denotes the processing time of the job in position $i$ on machine $m$. Similarly, $S_{[i, m]}, L_{[i, m]}$, and $U_{[i, m]}$ denote setup time, lower bound of setup time, and upper bound of setup time of the job in position $i$ on machine $m$, respectively. In contrast to $P_{j, m}$ and $P_{[i, m]}$ which are known before scheduling, exact values $S_{j, m}$ and $S_{[i, m]}$ are unknown.

For the deterministic versions of these problems where setup times are fixed (i.e., $S_{j, m}=L_{j, m}=U_{j, m}$ ), Yoshida and Hitomi [11] established the following formula for the completion time $C_{[j]}$ of the job in position $j$ :

$$
C_{[j]}=\max _{0 \leq u \leq j}\left[\sum_{i=1}^{u}\left(S_{[i, 1]}-S_{[i, 2]}+P_{[i, 1]}\right)-\sum_{i=1}^{u-1} P_{[i, 2]}\right]+\sum_{i=1}^{j}\left(S_{[i, 2]}+P_{[i, 2]}\right) .
$$

The above equation can be written as follows:

$$
\begin{aligned}
C_{[j]} & =\max _{0 \leq u \leq j}\left[\sum_{i=1}^{u}\left(S_{[i, 1]}+P_{[i, 1]}\right)-\sum_{i=1}^{u-1}\left(S_{[i, 2]}+P_{[i, 2]}\right)-S_{[u, 2]}\right]+\sum_{i=1}^{j}\left(S_{[i, 2]}+P_{[i, 2]}\right) \\
& =\max _{0 \leq u \leq j}\left[S P_{[u, 1]}-\left(S P_{[u-1,2]}+S_{[u, 2]}\right)\right]+S P_{[j, 2]},
\end{aligned}
$$

where we use notation $S P_{[j, m]}=\sum_{r=1}^{j}\left(S_{[r, m]}+P_{[r, m]}\right)$ for $j=1,2, \ldots, n$ and $m \in M=\{1,2\}$. Let $D_{j}=\max \left\{0, \sigma_{1}, \sigma_{2}, \ldots, \sigma_{j}\right\}$, where $\sigma_{j}=S P_{[j, 1]}-\left(S P_{[j-1,2]}+\right.$ $\left.S_{[j, 2]}\right), j=1,2, \ldots, n$, with the assumption that $S P_{[0,2]}=0$. Then, $C_{[j]}$ can be written as $C_{[j]}=S P_{[j, 2]}+D_{j}$. 
Once completion times of the $n$ jobs are known, then the makespan $C_{\max }$ and (TCT), are obtained as follows:

$$
\begin{aligned}
\mathrm{TCT} & =\sum_{j=1}^{n}\left(S P_{[j, 2]}+D_{j}\right), \\
C_{\max } & =S P_{[n, 2]}+D_{n} .
\end{aligned}
$$

It is clear from the latter equation that the term $S P_{[n, 2]}$ is a constant term which is independent of job sequence, and hence, minimization of $C_{\max }$ is equivalent to minimization of the term $D_{n}$, which denotes the total idle time on the second machine until all $n$ jobs are completed.

For each job $j \in J$ and machine $m \in M$, any feasible realization $S_{j, m}$ of setup time satisfies the inequalities $L_{j, m} \leq S_{j, m} \leq U_{j, m}$.

Before scheduling, we only know the lower and upper bounds of setup times given by the above inequalities, which define polytope (PT) of feasible vectors $S=\left(S_{1,1}, S_{1,2}, S_{2,1}, S_{2,2}, \ldots, S_{n, 1}, S_{n, 2}\right)$ of setup times as follows: PT $=\left\{S: L_{j, m} \leq\right.$ $\left.S_{j, m} \leq U_{j, m}, j \in J, m \in M\right\}$.

As it was mentioned so far, permutation schedules dominate schedules with different sequences of jobs on machine 1 and machine 2 for both problems under consideration. Therefore, we consider only the set of permutation schedules, and there are $n$ ! sequences (permutations) $Q=\left\{Q_{1}, Q_{2}, \ldots, Q_{n !}\right\}$ for the problems of $F 2\left|S_{j, m}, L_{j, m} \leq S_{j, m} \leq U_{j, m}\right| C_{\max }$ and $F 2 \mid S_{j, m}, L_{j, m} \leq S_{j, m} \leq$ $U_{j, m} \mid \sum C_{j, 2}$ that will be considered in finding the optimal sequence.

Similar to $[4,9]$ we use the following definition of a solution to the problems of $F 2\left|S_{j, m}, L_{j, m} \leq S_{j, m} \leq U_{j, m}\right| \sum C_{j}$ and $F 2\left|S_{j, m}, L_{j, m} \leq S_{j, m} \leq U_{j, m}\right| C_{\max }$. A set of sequences $Q^{*} \subseteq Q$ is a solution to the problem of $F 2 \mid S_{j, m}, L_{j, m} \leq$ $S_{j, m} \leq U_{j, m} \mid \sum C_{j}$ (or $F 2\left|S_{j, m}, L_{j, m} \leq S_{j, m} \leq U_{j, m}\right| C_{\max }$ ) if for each feasible vector $S \in \mathrm{PT}$ of setup times, the set $Q^{*}$ contains at least one optimal sequence. Thus, the whole set $Q$ of sequences is a trivial solution for the problems of $F 2\left|S_{j, m}, L_{j, m} \leq S_{j, m} \leq U_{j, m}\right| \sum C_{j}$ and $F 2\left|S_{j, m}, L_{j, m} \leq S_{j, m} \leq U_{j, m}\right| C_{\max }$. However, it is only possible to construct the whole set $Q$ for a small number of jobs. It is also impractical to choose the best sequence from a large set $Q^{*}$ of candidates as the processing of jobs evolves. Therefore, it is important to minimize the cardinality of solution $Q^{*}$ constructed for problems of $F 2\left|S_{j, m}, L_{j, m} \leq S_{j, m} \leq U_{j, m}\right| \sum C_{j}$ and $F 2\left|S_{j, m}, L_{j, m} \leq S_{j, m} \leq U_{j, m}\right| C_{\max }$. To this end, we introduce the following dominance relations on the set of sequences $Q$.

DeFinItion 2.1. For the problem $F 2\left|S_{j, m}, L_{j, m} \leq S_{j, m} \leq U_{j, m}\right| \sum C_{j}$, a sequence $Q_{1} \in Q$ dominates a sequence $Q_{2} \in Q$ with respect to PT if the inequality $\sum C_{i, m}\left(Q_{1}\right) \leq \sum C_{i, m}\left(Q_{2}\right)$ holds for any vector $S \in$ PT. Similarly, for the problem $F 2\left|S_{j, m}, L_{j, m} \leq S_{j, m} \leq U_{j, m}\right| C_{\max }$, a sequence $Q_{3} \in Q$ dominates a sequence $Q_{4} \in Q$ with respect to PT if the inequality $C_{\max }\left(Q_{3}\right) \leq C_{\max }\left(Q_{4}\right)$ holds for any vector $S \in \mathrm{PT}$. 
By Definition 2.1, a set of sequences $Q^{*} \subseteq Q$ is a solution to the problem $F 2\left|S_{j, m}, L_{j, m} \leq S_{j, m} \leq U_{j, m}\right| C_{\max }$ (or $F 2\left|S_{j, m}, L_{j, m} \leq S_{j, m} \leq U_{j, m}\right| \sum C_{j}$ ) if for each sequence $Q_{k} \in Q$, there exists a sequence from set $Q^{*}$ that dominates the sequence $Q_{k}$ with respect to PT.

3. Global dominance relations. Let $q_{h}$ denote a subsequence of a complete sequence $Q_{u} \in Q$ of all the $n$ jobs. Therefore, the notations of $Q_{1}=$ $\left(q_{1}, i, q_{2}, k, q_{3}\right)$ and $Q_{2}=\left(q_{1}, k, q_{2}, i, q_{3}\right)$ mean that the two sequences of $Q_{1}$ and $Q_{2}$ have the same jobs in all positions except that the jobs $i \in J$ and $k \in J$ are interchanged. When the jobs $i$ and $k$ are adjacent, such two complete sequences of $Q_{3}$ and $Q_{4}$ can be expressed as $Q_{3}=\left(q_{1}, i, k, q_{2}\right)$ and $Q_{4}=\left(q_{1}, k, i, q_{2}\right)$, respectively.

THEOREM 3.1. For the problem $F 2\left|S_{j, m}, L_{j, m} \leq S_{j, m} \leq U_{j, m}\right| C_{\max }$, the sequence $Q_{1}=\left(q_{1}, i, q_{2}, k, q_{3}\right) \in Q$ dominates the sequence $Q_{2}=\left(q_{1}, k, q_{2}, i, q_{3}\right) \in$ $Q$ with respect to $P T$ if the following two inequalities hold:

$$
\begin{aligned}
P_{k, 2} & \leq P_{i, 2}, \\
U_{i, 1}+P_{i, 1}+U_{k, 2} & \leq L_{k, 1}+P_{k, 1}+L_{i, 2} .
\end{aligned}
$$

THEOREM 3.2. For the problem $F 2\left|S_{j, m}, L_{j, m} \leq S_{j, m} \leq U_{j, m}\right| \sum C_{j}$, the sequence $Q_{1}=\left(q_{1}, i, q_{2}, k, q_{3}\right) \in Q$ dominates the sequence $Q_{2}=\left(q_{1}, k, q_{2}, i, q_{3}\right) \in$ $Q$ with respect to $P T$ if the following three inequalities hold:

$$
\begin{gathered}
P_{k, 2} \leq P_{i, 2}, \\
U_{i, 2}+P_{i, 2} \leq L_{k, 2}+P_{k, 2}, \\
U_{i, 1}+P_{i, 1}+U_{k, 2} \leq L_{k, 1}+P_{k, 1}+L_{i, 2} .
\end{gathered}
$$

Proofs of Theorems 3.1 And 3.2. We consider two job sequences $Q_{1}$ and $Q_{2}$, where $Q_{1}$ is a sequence in which job $i$ is in position $\alpha$ and job $k$ is in position $\beta$, where $\alpha<\beta$, whereas sequence $Q_{2}$ is obtained from sequence $Q_{1}$ by interchanging only the jobs in positions $\alpha$ and $\beta$. When necessary, we attach a corresponding sequence $Q_{u} \in Q$ to the notations $C_{[j]}, D_{j}, \sigma_{j}, S P_{[j, j]}$, and TCT introduced in Section 2, that is, we have $C_{[j]}\left(Q_{u}\right), D_{j}\left(Q_{u}\right), \sigma_{j}\left(Q_{u}\right)$, $S P_{[j, j]}\left(Q_{u}\right)$, and $\operatorname{TCT}\left(Q_{u}\right)$.

It should be clear that $S P_{[\alpha-1,1]}\left(Q_{1}\right)=S P_{[\alpha-1,1]}\left(Q_{2}\right)$ and $S P_{[\alpha-1,2]}\left(Q_{1}\right)=$ $S P_{[\alpha-1,2]}\left(Q_{2}\right)$ since both sequences have the same job in each position $1,2, \ldots$, $\alpha-1$. For $j=\alpha$, we obtain

$$
\begin{aligned}
& \sigma_{\alpha}\left(Q_{2}\right)=S P_{[\alpha-1,1]}\left(Q_{2}\right)+S_{k, 1}+P_{k, 1}-\left(S P_{[\alpha-1,2]}\left(Q_{2}\right)+S_{k, 2}\right), \\
& \sigma_{\alpha}\left(Q_{1}\right)=S P_{[\alpha-1,1]}\left(Q_{1}\right)+S_{i, 1}+P_{i, 1}-\left(S P_{[\alpha-1,2]}\left(Q_{1}\right)+S_{i, 2}\right) .
\end{aligned}
$$

From (3.3) we obtain

$$
\sigma_{\alpha}\left(Q_{1}\right) \leq \sigma_{\alpha}\left(Q_{2}\right)
$$


since $U_{i, 1}+P_{i, 1}+U_{k, 2} \leq L_{k, 1}+P_{k, 1}+L_{i, 2}$ always implies that $S_{i, 1}+P_{i, 1}+S_{k, 2} \leq$ $S_{k, 1}+P_{k, 1}+S_{i, 2}$.

For $j=\alpha+1, \alpha+2, \ldots, \beta-1$, we obtain

$$
\begin{aligned}
\sigma_{j}\left(Q_{2}\right)= & S P_{[\alpha-1,1]}\left(Q_{2}\right)+S_{k, 1}+P_{k, 1}+\sum_{r=\alpha+1}^{j}\left(S_{[r, 1]}+P_{[r, 1]}\right) \\
& -\left[S P_{[\alpha-1,2]}\left(Q_{2}\right)+S_{k, 2}+P_{k, 2}+\sum_{r=\alpha+1}^{j-1}\left(S_{[r, 2]}+P_{[r, 2]}\right)+S_{[j, 2]}\right], \\
\sigma_{j}\left(Q_{1}\right)= & S P_{[\alpha-1,1]}\left(Q_{1}\right)+S_{i, 1}+P_{i, 1}+\sum_{r=\alpha+1}^{j}\left(S_{[r, 1]}+P_{[r, 1]}\right) \\
& -\left[S P_{[\alpha-1,2]}\left(Q_{1}\right)+S_{i, 2}+P_{i, 2}+\sum_{r=\alpha+1}^{j-1}\left(S_{[r, 2]}+P_{[r, 2]}\right)+S_{[j, 2]}\right],
\end{aligned}
$$

where we assume that $\sum_{r=\alpha+1}^{\alpha}\left(S_{[r, 2]}+P_{[r, 2]}\right)=0$. Both sequences have the same job in all positions except for position $\alpha$, and hence, it follows that $\sigma_{j}\left(Q_{1}\right)-$ $\sigma_{j}\left(Q_{2}\right)=\left(S_{i, 1}+P_{i, 1}+S_{k, 2}+P_{k, 2}\right)-\left(S_{k, 1}+P_{k, 1}+S_{i, 2}+P_{i, 2}\right)$, which results in

$$
\sigma_{j}\left(Q_{1}\right) \leq \sigma_{j}\left(Q_{2}\right)
$$

since $P_{k, 2} \leq P_{i, 2}$ and $S_{i, 1}+P_{i, 1}+S_{k, 2} \leq S_{k, 1}+P_{k, 1}+S_{i, 2}$ (again this is implied by the fact that $\left.U_{i, 1}+P_{i, 1}+U_{k, 2} \leq L_{k, 1}+P_{k, 1}+L_{i, 2}\right)$.

For $j=\beta$, we obtain

$$
\begin{aligned}
\sigma_{\beta}\left(Q_{2}\right)= & S P_{[\alpha-1,1]}\left(Q_{2}\right)+S_{k, 1}+P_{k, 1}+\sum_{r=\alpha+1}^{\beta-1}\left(S_{[r, 1]}+P_{[r, 1]}\right)+S_{i, 1}+P_{i, 1} \\
& -\left[S P_{[\alpha-1,2]}\left(Q_{2}\right)+S_{k, 2}+P_{k, 2}+\sum_{r=\alpha+1}^{\beta-1}\left(S_{[r, 2]}+P_{[r, 2]}\right)+S_{i, 2}\right], \\
\sigma_{\beta}\left(Q_{1}\right)= & S P_{[\alpha-1,1]}\left(Q_{1}\right)+S_{i, 1}+P_{i, 1}+\sum_{r=\alpha+1}^{\beta-1}\left(S_{[r, 1]}+P_{[r, 1]}\right)+S_{k, 1}+P_{k, 1} \\
& -\left[S P_{[\alpha-1,2]}\left(Q_{1}\right)+S_{i, 2}+P_{i, 2}+\sum_{r=\alpha+1}^{\beta-1}\left(S_{[r, 2]}+P_{[r, 2]}\right)+S_{k, 2}\right] .
\end{aligned}
$$

It follows from (3.7) that

$$
\sigma_{\beta}\left(Q_{1}\right) \leq \sigma_{\beta}\left(Q_{2}\right)
$$

since $P_{k, 2} \leq P_{i, 2}$. It is obvious that $\sigma_{j}\left(Q_{1}\right)=\sigma_{j}\left(Q_{2}\right)$ for each $j=1,2, \ldots, \alpha-1$ since both sequences have the same job in these positions. It can easily be shown that $\sigma_{j}\left(Q_{1}\right)=\sigma_{j}\left(Q_{2}\right)$ for each $j=\beta+1, \beta+2, \ldots, n$. From these facts and (3.4), (3.6), and (3.8), we obtain

$$
\sigma_{j}\left(Q_{1}\right) \leq \sigma_{j}\left(Q_{2}\right)
$$


for each position $j=1,2, \ldots, n$. Therefore, $D_{n}\left(Q_{1}\right) \leq D_{n}\left(Q_{2}\right)$. This shows that the sequence $Q_{1}=\left(q_{1}, i, q_{2}, k, q_{3}\right) \in Q$ dominates the sequence $Q_{2}=$ $\left(q_{1}, k, q_{2}, i, q_{3}\right) \in Q$ with respect to $C_{\max }$. Observe that

$$
C_{[j]}\left(Q_{1}\right)=C_{[j]}\left(Q_{2}\right)
$$

for each position $j=1,2, \ldots, \alpha-1$ since both sequences have the same job in these positions. It is obvious that $D_{\alpha-1}\left(Q_{1}\right)=D_{\alpha-1}\left(Q_{2}\right)$. It can easily be shown that for each position $j=\beta, \beta+1, \ldots, n$,

$$
\begin{aligned}
C_{[j]}\left(Q_{1}\right)-C_{[j]}\left(Q_{2}\right)= & \max \left\{D_{\alpha-1}\left(Q_{1}\right), \sigma_{\alpha}\left(Q_{1}\right), \sigma_{\alpha+1}\left(Q_{1}\right), \ldots, \sigma_{j}\left(Q_{1}\right)\right\} \\
& -\max \left\{D_{\alpha-1}\left(Q_{2}\right), \sigma_{\alpha}\left(Q_{2}\right), \sigma_{\alpha+1}\left(Q_{2}\right), \ldots, \sigma_{j}\left(Q_{2}\right)\right\} .
\end{aligned}
$$

But it follows from (3.9) that

$$
\begin{aligned}
\max & \left\{D_{\alpha-1}\left(Q_{1}\right), \sigma_{\alpha}\left(Q_{1}\right), \sigma_{\alpha+1}\left(Q_{1}\right), \ldots, \sigma_{j}\left(Q_{1}\right)\right\} \\
& \leq \max \left\{D_{\alpha-1}\left(Q_{2}\right), \sigma_{\alpha}\left(Q_{2}\right), \sigma_{\alpha+1}\left(Q_{2}\right), \ldots, \sigma_{j}\left(Q_{2}\right)\right\},
\end{aligned}
$$

and therefore,

$$
C_{[j]}\left(Q_{1}\right) \leq C_{[j]}\left(Q_{2}\right)
$$

for each position $j=\beta, \beta+1, \ldots, n$. Observe that for $j=\alpha$,

$$
\begin{aligned}
& C_{[\alpha]}\left(Q_{2}\right)=S P_{[\alpha-1,2]}\left(Q_{2}\right)+S_{k, 2}+P_{k, 2}+\max \left\{D_{\alpha-1}\left(Q_{2}\right), \sigma_{\alpha}\left(Q_{2}\right)\right\}, \\
& C_{[\alpha]}\left(Q_{1}\right)=S P_{[\alpha-1,2]}\left(Q_{1}\right)+S_{i, 2}+P_{i, 2}+\max \left\{D_{\alpha-1}\left(Q_{1}\right), \sigma_{\alpha}\left(Q_{1}\right)\right\},
\end{aligned}
$$

and for $j=\alpha+1, \alpha+2, \ldots, \beta-1$,

$$
\begin{aligned}
C_{[j]}\left(Q_{2}\right)= & S P_{[\alpha-1,2]}\left(Q_{2}\right)+S_{k, 2}+P_{k, 2}+\sum_{r=\alpha+1}^{j}\left(S_{[r, 2]}+P_{[r, 2]}\right) \\
& +\max \left\{D_{\alpha-1}\left(Q_{2}\right), \sigma_{\alpha}\left(Q_{2}\right), \sigma_{\alpha+1}\left(Q_{2}\right), \ldots, \sigma_{j}\left(Q_{2}\right)\right\}, \\
C_{[j]}\left(Q_{1}\right)= & S P_{[\alpha-1,2]}\left(Q_{1}\right)+S_{i, 2}+P_{i, 2}+\sum_{r=\alpha+1}^{j}\left(S_{[r, 2]}+P_{[r, 2]}\right) \\
& +\max \left\{D_{\alpha-1}\left(Q_{1}\right), \sigma_{\alpha}\left(Q_{1}\right), \sigma_{\alpha+1}\left(Q_{1}\right), \ldots, \sigma_{j}\left(Q_{1}\right)\right\} .
\end{aligned}
$$

Hence, for each position $j=\alpha, \alpha+1, \ldots, \beta-1$, we obtain

$$
\begin{aligned}
C_{[j]}\left(Q_{1}\right)-C_{j}\left(Q_{2}\right) & \\
= & \left(S_{i, 2}+P_{i, 2}\right)-\left(S_{k, 2}+P_{k, 2}\right) \\
& +\max \left\{D_{\alpha-1}\left(Q_{1}\right), \ldots, \sigma_{j}\left(Q_{1}\right)\right\}-\max \left\{D_{\alpha-1}\left(Q_{2}\right), \ldots, \sigma_{j}\left(Q_{2}\right)\right\} .
\end{aligned}
$$

However, $U_{i, 2}+P_{i, 2} \leq L_{k, 2}+P_{k, 2}$ implies $S_{i, 2}+P_{i, 2} \leq S_{k, 2}+P_{k, 2}$ and, by (3.12),

$$
\max \left\{D_{\alpha-1}\left(Q_{1}\right), \ldots, \sigma_{j}\left(Q_{1}\right)\right\} \leq \max \left\{D_{\alpha-1}\left(Q_{2}\right), \ldots, \sigma_{j}\left(Q_{2}\right)\right\},
$$


and therefore,

$$
C_{[j]}\left(Q_{1}\right) \leq C_{[j]}\left(Q_{2}\right)
$$

for each $j=\alpha, \alpha+1, \ldots, \beta-1$. Now it follows from (3.10), (3.13), and (3.18) that the sequence $Q_{1}=\left(q_{1}, i, q_{2}, k, q_{3}\right) \in Q$ dominates the sequence $Q_{2}=$ $\left(q_{1}, k, q_{2}, i, q_{3}\right) \in Q$ with respect to TCT minimization. This completes the proofs.

REMARK 3.3. Observe that the sufficient conditions given in Theorem 3.1 are included in those of Theorem 3.2. Thus, using the latter conditions for some flowshop problems, one can minimize both objective functions $C_{\max }$ and $\sum C_{j}$.

4. Local dominance relations. In this section, we prove similar sufficient conditions for dominance relations for the case of adjacent jobs.

THEOREM 4.1. For the problem $F 2\left|S_{j, m}, L_{j, m} \leq S_{j, m} \leq U_{j, m}\right| C_{\max }$, the sequence $Q_{3}=\left(q_{1}, i, k, q_{2}\right) \in Q$ dominates the sequence $Q_{4}=\left(q_{1}, k, i, q_{2}\right) \in Q$ with respect to $P T$ if the inequality $U_{i, 1}+P_{i, 1}+U_{k, 2} \leq L_{k, 1}+P_{k, 1}+L_{i, 2}$ and one of the following inequalities hold:

(i) $U_{k, 1}+P_{k, 1} \leq P_{i, 2}+L_{k, 2}$,

(ii) $U_{i, 1}+P_{i, 1} \leq L_{i, 2}+P_{i, 2}$,

(iii) $P_{k, 2} \leq P_{i, 2}$.

THEOREM 4.2. For the problem $F 2\left|S_{j, m}, L_{j, m} \leq S_{j, m} \leq U_{j, m}\right| \sum C_{i}$, the sequence $Q_{3}=\left(q_{1}, i, k, q_{2}\right) \in Q$ dominates the sequence $Q_{4}=\left(q_{1}, k, i, q_{2}\right) \in Q$ with respect to $P$ T if the inequalities $U_{i, 1}+P_{i, 1}+U_{k, 2} \leq L_{k, 1}+P_{k, 1}+L_{i, 2}, U_{i, 2}+P_{i, 2} \leq$ $L_{k, 2}+P_{k, 2}$ and one of the following inequalities hold:

(i) $U_{k, 1}+P_{k, 1} \leq P_{i, 2}+L_{k, 2}$,

(ii) $U_{i, 1}+P_{i, 1} \leq L_{i, 2}+P_{i, 2}$,

(iii) $P_{k, 2} \leq P_{i, 2}$.

Proofs OF TheOrems 4.1 AND 4.2. We assume that the sequence $Q_{3}$ has job $i \in J$ in position $\alpha$ and job $k \in J$ in position $\alpha+1$, and the sequence $Q_{4}$ is obtained from the sequence $Q_{3}$ by interchanging only the two jobs in positions $\alpha$ and $\alpha+1$. For these two sequences, we obtain

$$
\begin{aligned}
\sigma_{\alpha}\left(Q_{3}\right)= & S P_{[\alpha-1,1]}\left(Q_{3}\right)+S_{i, 1}+P_{i, 1}-S P_{[\alpha-1,2]}\left(Q_{3}\right)-S_{i, 2}, \\
\sigma_{\alpha}\left(Q_{4}\right)= & S P_{[\alpha-1,1]}\left(Q_{4}\right)+S_{k, 1}+P_{k, 1}-S P_{[\alpha-1,2]}\left(Q_{4}\right)-S_{k, 2}, \\
\sigma_{\alpha+1}\left(Q_{3}\right)= & S P_{[\alpha-1,1]}\left(Q_{3}\right)+S_{i, 1} \\
& +P_{i, 1}+S_{k, 1}+P_{k, 1}-S P_{[\alpha-1,2]}\left(Q_{3}\right)-S_{i, 2}-P_{i, 2}-S_{k, 2}, \\
\sigma_{\alpha+1}\left(Q_{4}\right)= & S P_{[\alpha-1,1]}\left(Q_{4}\right)+S_{k, 1} \\
& +P_{k, 1}+S_{i, 1}+P_{i, 1}-S P_{[\alpha-1,2]}\left(Q_{4}\right)-S_{k, 2}-P_{k, 2}-S_{i, 2} .
\end{aligned}
$$


Since both sequences have the same jobs in positions $1,2, \ldots, \alpha-1$, we obtain the equality $D_{\alpha-1}\left(Q_{3}\right)=D_{\alpha-1}\left(Q_{4}\right)$. Let $D_{\alpha-1}=D_{\alpha-1}\left(Q_{3}\right)=D_{\alpha-1}\left(Q_{4}\right)$. Then we have

$$
\begin{aligned}
C_{[\alpha]}\left(Q_{3}\right)= & S P_{[\alpha-1,2]}\left(Q_{3}\right)+S_{i, 2}+P_{i, 2}+\max \left\{D_{\alpha-1}, \sigma_{\alpha}\left(Q_{3}\right)\right\}, \\
C_{[\alpha]}\left(Q_{4}\right)= & S P_{[\alpha-1,2]}\left(Q_{4}\right)+S_{k, 2}+P_{k, 2}+\max \left\{D_{\alpha-1}, \sigma_{\alpha}\left(Q_{4}\right)\right\}, \\
C_{[\alpha+1]}\left(Q_{3}\right)= & S P_{[\alpha-1,2]}\left(Q_{3}\right)+S_{i, 2}+P_{i, 2}+S_{k, 2}+P_{k, 2} \\
& +\max \left\{D_{\alpha-1}, \sigma_{\alpha}\left(Q_{3}\right), \sigma_{\alpha+1}\left(Q_{3}\right)\right\}, \\
C_{[\alpha+1]}\left(Q_{4}\right)= & S P_{[\alpha-1,2]}\left(Q_{4}\right)+S_{k, 2}+P_{k, 2}+S_{i, 2}+P_{i, 2} \\
& +\max \left\{D_{\alpha-1}, \sigma_{\alpha}\left(Q_{4}\right), \sigma_{\alpha+1}\left(Q_{4}\right)\right\} .
\end{aligned}
$$

Observe that $S P_{[\alpha-1,1]}\left(Q_{3}\right)=S P_{[\alpha-1,1]}\left(Q_{4}\right)$ and $S P_{[\alpha-1,2]}\left(Q_{3}\right)=S P_{[\alpha-1,2]}\left(Q_{4}\right)$ because both sequences have the same jobs in positions $1,2, \ldots, \alpha-1$. As a result, it holds that

$$
\sigma_{j}\left(Q_{3}\right)=\sigma_{j}\left(Q_{4}\right)
$$

for each position $j=1,2, \ldots, \alpha-1$. It can easily be shown that $\sigma_{j}\left(Q_{3}\right)=\sigma_{j}\left(Q_{4}\right)$ for each position $j=\alpha+2, \alpha+3, \ldots, n$. It follows from the above equalities that

$$
\sigma_{\alpha}\left(Q_{3}\right) \leq \sigma_{\alpha}\left(Q_{4}\right)
$$

since $U_{i, 1}+P_{i, 1}+U_{k, 2} \leq L_{k, 1}+P_{k, 1}+L_{i, 2}$. From (4.4),

$$
\max \left\{D_{\alpha-1}, \sigma_{\alpha}\left(Q_{3}\right)\right\} \leq \max \left\{D_{\alpha-1}, \sigma_{\alpha}\left(Q_{4}\right)\right\} .
$$

Again from the above inequalities if $U_{k, 1}+P_{k, 1} \leq P_{i, 2}+L_{k, 2}$, then

$$
\sigma_{\alpha+1}\left(Q_{3}\right) \leq \sigma_{\alpha}\left(Q_{3}\right),
$$

if $U_{i, 1}+P_{i, 1} \leq L_{i, 2}+P_{i, 2}$, then

$$
\sigma_{\alpha+1}\left(Q_{3}\right) \leq \sigma_{\alpha}\left(Q_{4}\right)
$$

and if $P_{k, 2} \leq P_{i, 2}$, then

$$
\sigma_{\alpha+1}\left(Q_{3}\right) \leq \sigma_{\alpha+1}\left(Q_{4}\right)
$$

If any one of the inequalities (4.6), (4.7), and (4.8) holds, then

$$
\max \left\{D_{\alpha-1}, \sigma_{\alpha}\left(Q_{3}\right), \sigma_{\alpha+1}\left(Q_{3}\right)\right\} \leq \max \left\{D_{\alpha-1}, \sigma_{\alpha}\left(Q_{4}\right), \sigma_{\alpha+1}\left(Q_{4}\right)\right\} .
$$

Therefore, from (4.3), (4.4), and (4.9), we obtain $D_{n}\left(Q_{3}\right) \leq D_{n}\left(Q_{4}\right)$, which shows that the sequence $Q_{3}=\left(q_{1}, i, k, q_{2}\right) \in Q$ dominates the sequence $Q_{4}=$ $\left(q_{1}, k, i, q_{2}\right) \in Q$ for criterion of minimizing $C_{\max }$. 
Taking the difference between completion times of the jobs in positions $\alpha$ and $\alpha+1$ for the two sequences yields

$$
\begin{aligned}
& {\left[C_{[\alpha]}\left(Q_{3}\right)+C_{[\alpha+1]}\left(Q_{3}\right)\right]-\left[C_{[\alpha]}\left(Q_{4}\right)+C_{[\alpha+1]}\left(Q_{4}\right)\right] } \\
&=\left(S_{i, 2}+P_{i, 2}\right)-\left(S_{k, 2}+P_{k, 2}\right) \\
&+\max \left\{D_{\alpha-1}, \sigma_{\alpha}\left(Q_{3}\right)\right\}-\max \left\{D_{\alpha-1}, \sigma_{\alpha}\left(Q_{4}\right)\right\} \\
&+\max \left\{D_{\alpha-1}, \sigma_{\alpha}\left(Q_{3}\right), \sigma_{\alpha+1}\left(Q_{3}\right)\right\}-\max \left\{D_{\alpha-1}, \sigma_{\alpha}\left(Q_{4}\right), \sigma_{\alpha+1}\left(Q_{4}\right)\right\} .
\end{aligned}
$$

From (4.5), (4.9), and the fact that $U_{i, 2}+P_{i, 2} \leq L_{k, 2}+P_{k, 2}$, we obtain

$$
C_{[\alpha]}\left(Q_{3}\right)+C_{[\alpha+1]}\left(Q_{3}\right) \leq C_{[\alpha]}\left(Q_{4}\right)+C_{[\alpha+1]}\left(Q_{4}\right) .
$$

It can also be shown that for each position $j=\alpha+2, \alpha+3, \ldots, n$,

$$
\begin{aligned}
C_{[j]}\left(Q_{3}\right)-C_{[j]}\left(Q_{4}\right) & \\
= & \max \left\{D_{\alpha-1}, \sigma_{\alpha}\left(Q_{3}\right), \sigma_{\alpha+1}\left(Q_{3}\right), \sigma_{\alpha+2}\left(Q_{3}\right), \ldots, \sigma_{j}\left(Q_{3}\right)\right\} \\
& -\max \left\{D_{\alpha-1}, \sigma_{\alpha}\left(Q_{4}\right), \sigma_{\alpha+1}\left(Q_{4}\right), \sigma_{\alpha+2}\left(Q_{4}\right), \ldots, \sigma_{j}\left(Q_{4}\right)\right\} .
\end{aligned}
$$

We observe that $\sigma_{k}\left(Q_{3}\right)=\sigma_{k}\left(Q_{4}\right)$ for each $k=\alpha+2, \alpha+3, \ldots, n$. Therefore, from the inequality of (4.9) we obtain

$$
C_{[j]}\left(Q_{3}\right) \leq C_{[j]}\left(Q_{4}\right)
$$

for each position $j=\alpha+2, \alpha+3, \ldots, n$. It is obvious that

$$
C_{[j]}\left(Q_{3}\right)=C_{[j]}\left(Q_{4}\right)
$$

for each $j=1,2, \ldots, \alpha-1$. Clearly, from (4.5), (4.9), (4.11), (4.13), and (4.14), we obtain the inequality $\operatorname{TCT}\left(Q_{3}\right) \leq \operatorname{TCT}\left(Q_{4}\right)$ which completes the proofs.

Remark 3.3 is valid for Theorems 4.1 and 4.2 as well.

5. Examples. Let $n=4$ and let numerical input data for both examples of problems $F 2\left|S_{j, m}, L_{j, m} \leq S_{j, m} \leq U_{j, m}\right| C_{\max }$ and $F 2\left|S_{j, m}, L_{j, m} \leq S_{j, m} \leq U_{j, m}\right| \sum C_{j}$ be given in Table 5.1 .

For problem $F 2\left|S_{j, m}, L_{j, m} \leq S_{j, m} \leq U_{j, m}\right| C_{\max }$, Theorem 3.1 implies the following precedence relations in an optimal sequence for a feasible vector $S \in \mathrm{PT}$ of setup times, polytope PT being defined in Table 5.1. Job 1 precedes job 3 and job 4 . Job 2 precedes job 3 and job 4 . Job 3 precedes job 4 . The order of job 1 and job 2 is not defined in an optimal sequence for the above example of problem $F 2\left|S_{j, m}, L_{j, m} \leq S_{j, m} \leq U_{j, m}\right| C_{\max }$ (both Theorems 3.1 and 4.1 do not define the order of jobs 1 and 2). Thus, the two sequences $Q_{1}=$ $(1,2,3,4)$ and $Q_{2}=(2,1,3,4)$ dominate all sequences from the set $Q$ with respect to PT, and we obtain the solution $Q^{*}=\left\{Q_{1}, Q_{2}\right\}$ for the example of 
TABLE 5.1. Job processing times, lower bounds, and upper bounds of setup times.

\begin{tabular}{c|crrrrr}
\hline Job $j$ & $L_{j, 1}$ & $U_{j, 1}$ & $P_{j, 1}$ & $L_{j, 2}$ & $U_{j, 2}$ & $P_{j, 2}$ \\
\hline$j=1$ & 1 & 2 & 4 & 4 & 6 & 12 \\
$j=2$ & 2 & 4 & 5 & 6 & 8 & 10 \\
$j=3$ & 2 & 4 & 8 & 4 & 6 & 10 \\
$j=4$ & 9 & 10 & 12 & 11 & 12 & 5 \\
\hline
\end{tabular}

problem $F 2\left|S_{j, m}, L_{j, m} \leq S_{j, m} \leq U_{j, m}\right| C_{\max }$ with numerical input data defined in Table 5.1.

It is easy to see that this solution $Q^{*}$ is minimal. Indeed, there exists vector $S_{1}=(1,6,4,6,2,4,9,11) \in \mathrm{PT}$ for which sequence $Q_{1}$ is optimal (for vector $S_{1}$ of setup times, the second machine has no idle time with sequence $Q_{1}$ of four jobs, and $\left.C_{\max }\left(Q_{1}\right)=64\right)$, while sequence $Q_{2}$ is not optimal $\left(C_{\max }\left(Q_{2}\right)=67\right.$ for vector $S_{1}$ of setup times).

On the other hand, there exists vector $S_{2}=(2,4,2,8,2,4,9,11) \in$ PT for which sequence $Q_{2}$ is optimal (for vector $S_{2}$ of setup times, the second machine has no idle time with sequence $Q_{2}$ of four jobs, and $C_{\max }\left(Q_{2}\right)=64$ ), while sequence $Q_{1}$ is not optimal ( $C_{\max }\left(Q_{1}\right)=66$ for vector $S_{2}$ of setup times).

For the above example of problem $F 2\left|S_{j, m}, L_{j, m} \leq S_{j, m} \leq U_{j, m}\right| \sum C_{j}$, Theorem 3.2 shows that for any feasible setup times, there exists optimal sequence of four jobs in which job 3 precedes job 4 . The order of other jobs from the set $J=\{1,2,3,4\}$ is defined via neither Theorem 3.2 nor Theorem 4.2. Thus, instead of considering $4 !=24$, sequences $Q$ of four jobs, it is sufficient to consider 12 sequences with job 3 preceding job 4 .

6. Conclusions. In this paper, we relax the assumption that setup times are deterministic and consider setup times to be random variables with free distributions where only their lower and upper bounds are given. We develop both local and global dominance relations for the problems of $F 2 \mid S_{j, m}, L_{j, m} \leq S_{j, m} \leq$ $U_{j, m} \mid C_{\max }$ and $F 2\left|S_{j, m}, L_{j, m} \leq S_{j, m} \leq U_{j, m}\right| \sum C_{j}$. In many cases, these relations allow to decrease the cardinality of solution $Q^{*}$ which has to be constructed for both problems to have a guarantee that set $Q^{*}$ contains at least one optimal job sequence for each feasible realization of setup times. Testing all the conditions presented in Theorems 3.1, 3.2, 4.1, and 4.2 takes a polynomial time.

Similar approach may be used for larger number of machines $(m>2)$ in a flowshop problem under uncertainty of setup times and for other generalizations of problems $F 2\left|S_{j, m}, L_{j, m} \leq S_{j, m} \leq U_{j, m}\right| C_{\max }$ and $F 2 \mid S_{j, m}, L_{j, m} \leq$ $S_{j, m} \leq U_{j, m} \mid \sum C_{j}$. Another interesting question for future research is to study sequence-dependent random and bounded setup times, which are more complicated. 
ACKNOWLEDGMENTS. The research of the first two authors was supported by Kuwait University Research Administration project EM01/01 and that of the third author was supported by INTAS project 00-217 and Fundamental Research Fund of Belarus project 99-119.

\section{REFERENCES}

[1] A. Allahverdi, Two-stage production scheduling with separated set-up times and stochastic breakdowns, J. Oper. Res. Soc. 46 (1995), no. 7, 896-904.

[2] _ Minimizing mean flowtime in a two-machine flowshop with sequenceindependent setup times, Comput. Oper. Res. 27 (2000), no. 2, 111-127.

[3] A. Allahverdi, J. N. D. Gupta, and T. Aldowiasan, A review of scheduling research involving setup considerations, OMEGA The International Journal of Management Science 27 (1999), no. 2, 219-239.

[4] A. Allahverdi and Y. Sotskov, Two-machine flowshop minimum-length scheduling problem with random and bounded processing times, Int. Trans. Oper. Res. 10 (2003), no. 1, 65-76.

[5] P. C. Bagga and K. Khurana, Two-machine flowshop with separated sequenceindependent setup times: mean completion time criterion, Indian J. Management Systems 2 (1986), 47-57.

[6] T. Gonzalez and S. Sahni, Flowshop and jobshop schedules: complexity and approximation, Operations Res. 26 (1978), no. 1, 36-52.

[7] S. M. Johnson, Optimal two- and three-stage production schedules with setup times included, Naval Res. Logist. Quart. 1 (1954), 61-68.

[8] K. Khurana and P. C. Bagga, Minimizing the makespan in a 2-machine flowshop with time lags and setup conditions, Z. Oper. Res. Ser. A-B 28 (1984), no. 5, A163-A174.

[9] T.-C. Lai and Y. N. Sotskov, Sequencing with uncertain numerical data for makespan minimisation, J. Oper. Res. Soc. 50 (1999), 230-243.

[10] T.-C. Lai, Y. N. Sotskov, N. Y. Sotskova, and F. Werner, Optimal makespan scheduling with given bounds of processing times, Math. Comput. Modelling 26 (1997), no. 3, 67-86.

[11] T. Yoshida and K. Hitomi, Optimal two-stage production scheduling with setup times separated, AIIE Trans. 11 (1979), 261-263.

Ali Allahverdi: Department of Industrial and Management Systems Engineering, College of Engineering and Petroleum, Kuwait University, P. O. Box 5969, Safat, Kuwait

E-mail address: a11ahverdi@kuc01. kuniv.edu. kw

Tariq Aldowaisan: Department of Industrial and Management Systems Engineering, College of Engineering and Petroleum, Kuwait University, P. O. Box 5969, Safat, Kuwait

E-mail address: tariq@kuc01.kuniv.edu.kw

Yuri N. Sotskov: Institute of Engineering Cybernetics, 6 Surganova Street, 220012 Minsk, Belarus

E-mail address: sotskov@newman . bas-net. by 


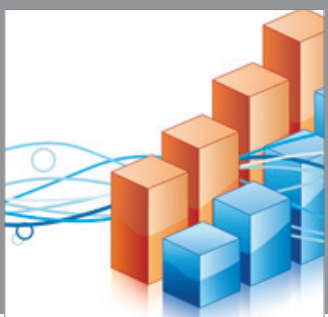

Advances in

Operations Research

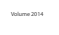

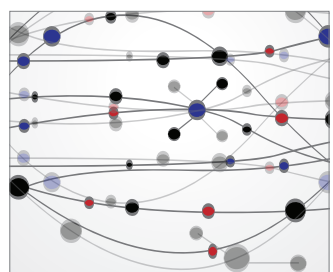

\section{The Scientific} World Journal
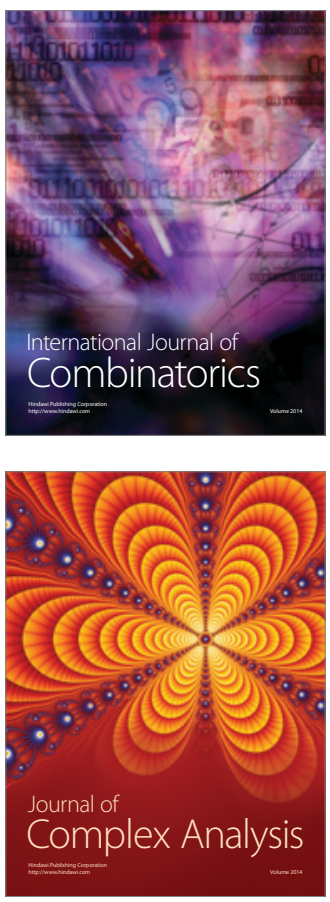

International Journal of

Mathematics and

Mathematical

Sciences
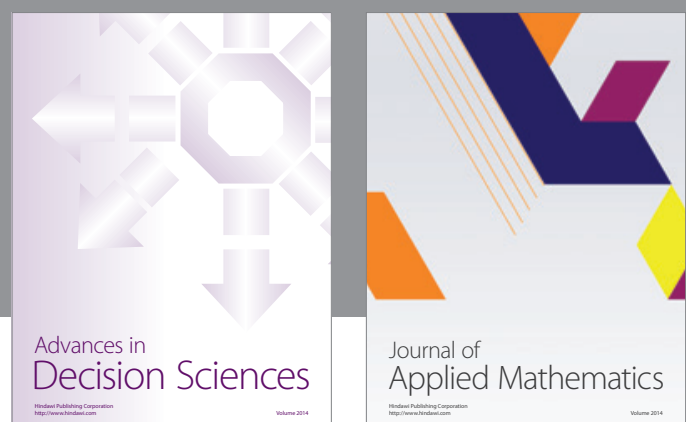

Journal of

Applied Mathematics
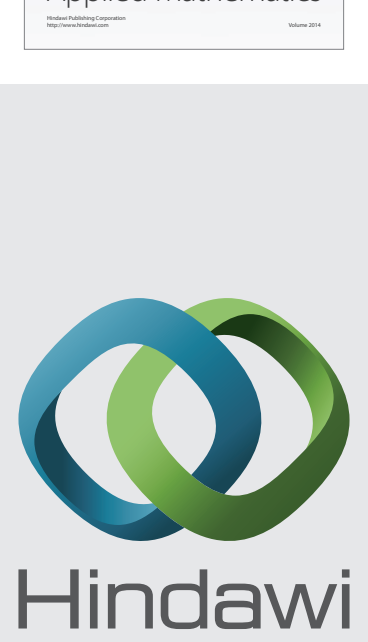

Submit your manuscripts at http://www.hindawi.com
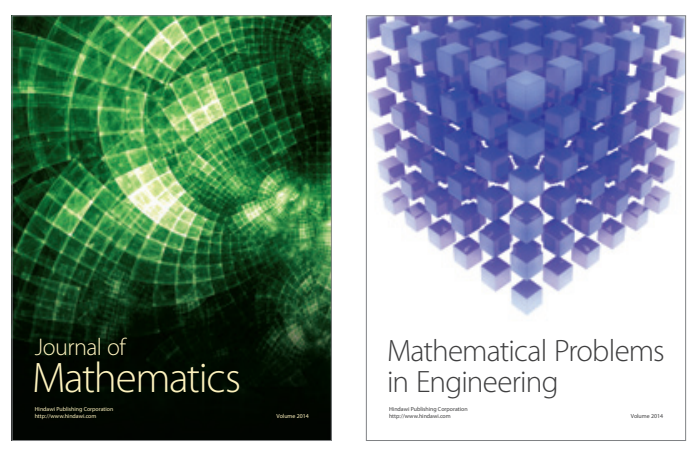

Mathematical Problems in Engineering
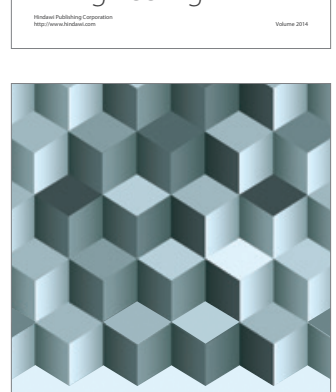

Journal of

Function Spaces
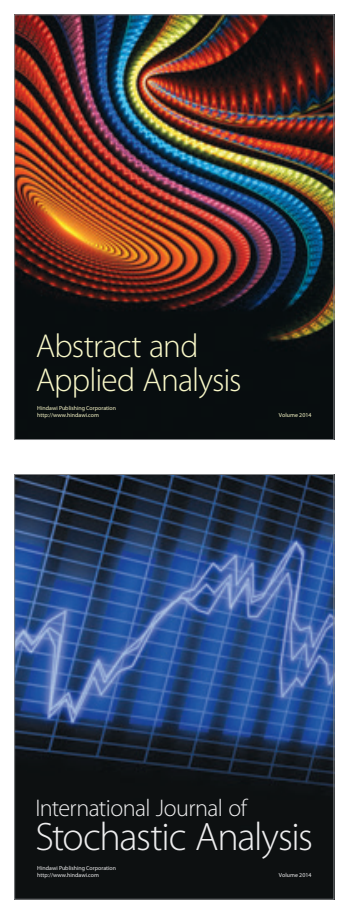

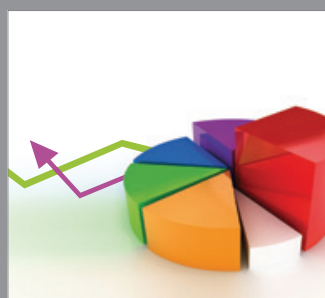

ournal of

Probability and Statistics

Promensencen
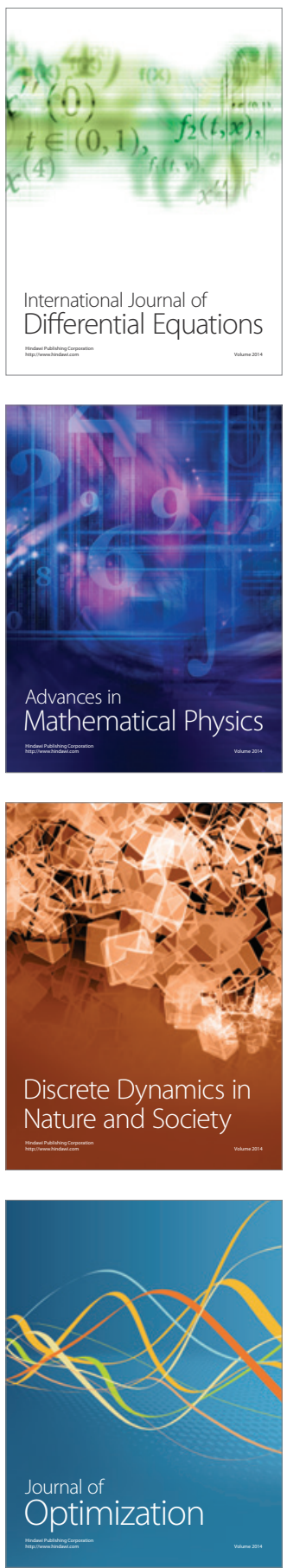